\title{
Takayasu's arteritis presenting with common carotid artery dissection: A rare case report
}

\author{
RUONAN HAO ${ }^{1}$, JIE ZHANG ${ }^{1}$, ZHICHENG MA ${ }^{2}$, MING XIAO ${ }^{3}$, \\ LEI ZHOU ${ }^{4}$, NAN KANG ${ }^{1}$, XIAOYU LIANG ${ }^{1}$ and FUXIN LI ${ }^{1}$ \\ ${ }^{1}$ Department of General Surgery, General Hospital, Tianjin Medical University, Tianjin 300052; \\ ${ }^{2}$ Department of General Surgery, Tianjin First Center Hospital, Tianjin 300192; \\ Departments of ${ }^{3}$ Cardiac Surgery and ${ }^{4}$ Rheumatism, General Hospital, Tianjin Medical University, Tianjin 300052, P.R. China
}

Received May 5, 2015; Accepted June 2, 2016

DOI: 10.3892/etm.2016.3861

\begin{abstract}
Takayasu's arteritis (TA) is a rare, idiopathic, chronic inflammatory disease associated with cell-mediated inflammation and involving predominantly the aorta and its primary branches. TA results in stenosis, occlusion or aneurysmal degeneration of large arteries pathologically. However, the incidence of dissection in arteries is low. As one of the primary causes of aortic dissection-associated ischemic stroke in young adults, only a limited number of TA cases presenting with aortic dissection have been published to date. In the present study, a case of common carotid artery dissection in a young patient with TA, without evident clinical manifestations, is presented. This is a rare clinical phenomenon, but may be a risk factor of acute cerebrovascular events. Clinical examination and satisfactory imaging are crucial for the correct diagnosis and management of patients with TA with dissection in arteries, and great attention is required in order to prevent the occurrence of cerebrovascular ischemia.
\end{abstract}

\section{Introduction}

Takayasu's arteritis (TA) is a chronic inflammatory disease with an unknown origin, which usually affects the aorta and its primary branches. TA typically occurs prior to the age of 40 years and has a morbidity of $0.6 \%$ (1). The aetiopathogenesis of the disease has not been clarified yet. Infections, autoimmunity and genetic factors have been examined as aetiologic factors. The treatment of TA begins with pharmacological control of the acute arteritis in order to induce clinical remission, followed by treatment of vascular abnormalities (2). The arteries suffering from TA are characterized by the

Correspondence to: Dr Jie Zhang, Department of General Surgery, General Hospital, Tianjin Medical University, 154 Anshan Road, Heping, Tianjin 300052, P.R. China

E-mail: wxq_bf@163.com

Key words: Takayasu's arteritis, dissection, inflammation, stenosis, ischemia proliferation of intima, focal leukocytic infiltration of the tunica media and degeneration of the external elastic lamina, leading to vessel wall thickening, vessel stenosis and occlusion, which are the most common presenting features $(3,4)$. However, aneurysmal dilatations occur less frequently (2) and arterial dissection is considerably rare (5), can produce a wide range of symptoms and typically presents with severe chest or back pain (6). Aortic dissection in the common carotid artery is an unusual pathologic characteristic of TA and has been rarely reported (7). The present study describes an atypical case of chronic aortic dissection in the common carotid artery in a patient with TA.

\section{Case report}

A 25-year-old male patient with a history of TA for 10 years, and had received glucocorticoid treatment, was referred to the General Hospital, Tinjin Medical University (Tianjin, China) in December 2014 due to chest tightness, shortness of breath and hemoptysis, without fever, fatigue or headaches. On admission, clinical examination was unremarkable, aside from increased heart rate of $115 \mathrm{bpm}$ and elevated blood pressure of 192/100 mmHg. There was no appreciable discrepancy in the blood pressure between the arms and no sign of acute limb ischemia, while all the peripheral pulses of the patient were present. Echocardiography revealed heart failure, indicated by a decreased ejection fraction and cardiac index. Lung and abdominal examination findings were normal, and no abnormality was reported in the computed tomography and magnetic resonance angiography scans of the cerebrovascular and neurologic systems. Hematological analyses exhibited an elevated erythrocyte sedimentation rate (ESR) of $37 \mathrm{~mm} / \mathrm{h}$ (normal value, $0-15 \mathrm{~mm} / \mathrm{h}$ ) and serum C-reactive protein level of $7.79 \mathrm{mg} / \mathrm{dl}$ (normal value, $0-0.8 \mathrm{mg} / \mathrm{dl}$ ). On carotid sonography, the patient's bilateral common carotid arteries presented mild long-segment, homogeneous, circumferential wall thickening, as well as a decrease in lumen diameter (left, 35\% stenosis; right, $43 \%$ stenosis) and dissection in the left common carotid artery (Fig. 1A). On color Doppler sonography, the dissected artery was filled with blood and the peak systolic velocity (PSV) of the artery was as high as $305 \mathrm{~cm} / \mathrm{sec}$ (normal range, $91.3 \pm 20.7 \mathrm{~cm} / \mathrm{sec}$ ). Additionally, contrast-enhanced ultrasound 
A

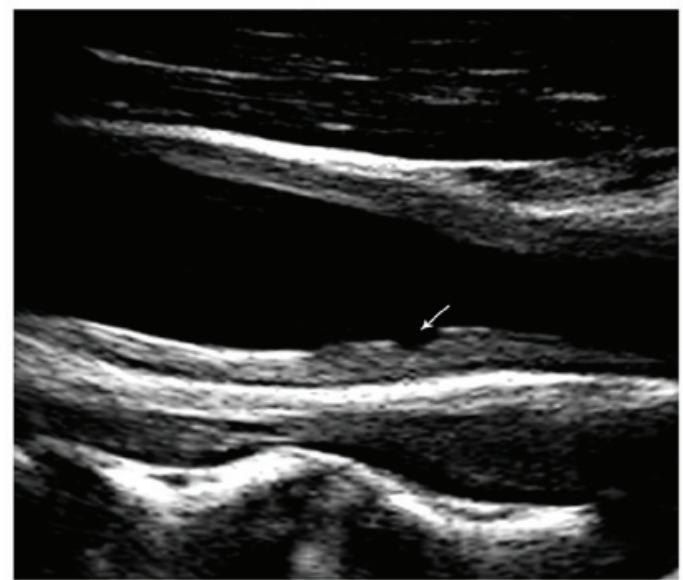

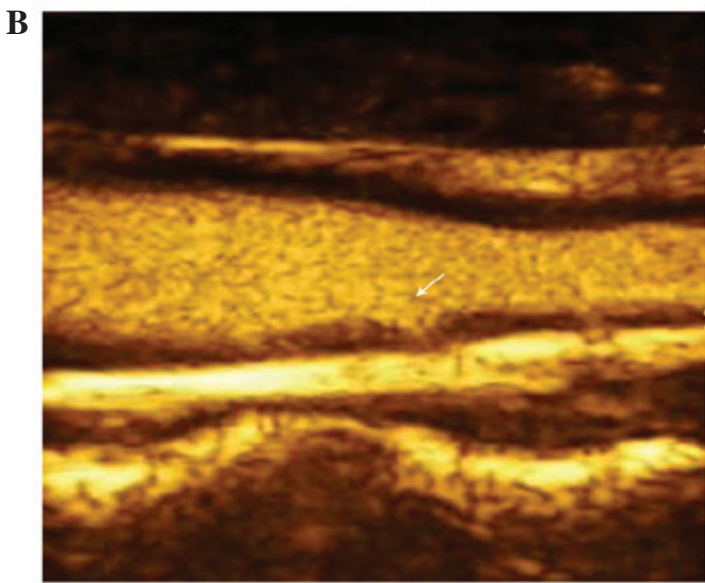

Figure 1. (A) Carotid sonography showed common carotid artery long-segment wall thickening and an intimal dissection. (B) Contrast-enhanced ultrasound showed uniform enhancement of the contrast agent between the dissection and intravascular. The arrow indicates artery dissection.
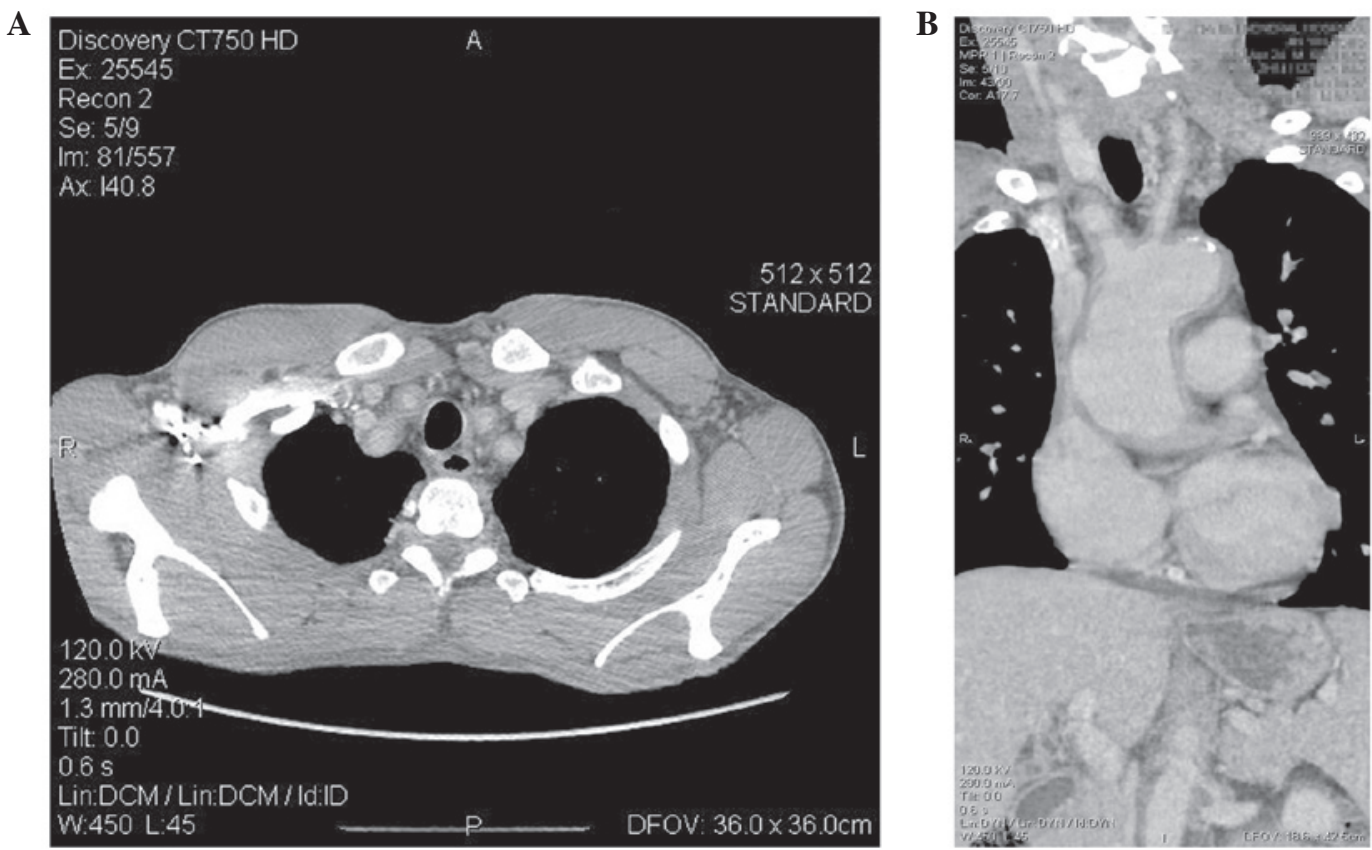

Figure 2. Computed tomographic angiography presented a (A) cross sectional and (B) coronal view of diffuse wall thickening of common carotid arteries and a decrease in the lumen diameter.

examination with sulfur hexafluoride $(1.5 \mathrm{ml}$; Bracco Group, Milan, Italy) showed uniform enhancement of contrast agent between the dissection and intravascular (Fig. 1B). For evaluation of the systemic large-artery involvement, computed tomographic angiography using ioversol contrast agent $(20 \mathrm{ml}$; Jiangsu Hengrui Medicine Co., Lianyungang, China) was performed and revealed diffuse wall thickening and stenosis of the left subclavian artery, common carotid arteries, right brachiocephalic trunk, diaphragm aorta, abdominal aorta, renal arteries and superior mesenteric arteries (Fig. 2A and B). A diagnosis was made, concluding TA involving the left subclavian artery, common carotid arteries, right brachiocephalic trunk, diaphragm aorta, abdominal aorta, renal arteries and superior mesenteric arteries.

The patient was treated with furosemide $(20 \mathrm{mg}$ daily for 5 days followed by dosage adjustments; Zhaohui
Pharmaceutical Co., Ltd., Shanghai, China), nifedipine (20 mg twice daily; Talison Pharmaceutical Co., Ltd., Zhejiang, China), prednisone (1 $\mathrm{mg} / \mathrm{kg}$ daily, gradually reduced; Xinyi Pharmaceutical Co., Ltd., Shanghai, China) and cyclophosphamide (2 mg daily, gradually reduced; Jin Shi Pharmaceutical Co., Ltd., Tianjin, China) and no surgical interventions were performed. The symptoms of chest tightness and shortness of breath were resolved after three days following admission and hypertension was controlled. After the patient was discharged, follow-up once every two months within six months was performed, followed by 6 monthly visit to hospital. During the follow-up period, there was no obvious clinical symptoms, and the patient remained on steroid treatment combined with immunosuppressive therapy. Written informed patient consent was obtained in the present study. 


\section{Discussion}

TA is a rare clinical condition that is characterized by chronic panarteritis of the aorta and its primary branches, including the subclavian, common carotid, coronary and renal arteries, and may result in localized stenoses, vascular occlusion, dilatation and aneurysm formation (8). TA induces a variety of non-specific inflammatory systemic signs and symptoms, such as fever, fatigue, headaches, arthralgia, malaise and weight loss in the chronic phase (9). In addition, it is characterized by marked thickening of the aortic wall, with fibrosis of the intima and adventitia. The thickening of the entire vessel wall results in stiffness, and this is the primary sign identified using imaging (10).

In the present study, the patient's clinical symptoms were not evident, although his ESR was raised; this indicated inflammatory activity. Chest tightness, shortness of breath and hemoptysis may also be caused by heart failure. Color Doppler sonography demonstrated homogeneous, mid-echoic, circumferential wall thickening of the common carotid artery, previously described as the 'macaroni sign', which is a pathognomonic sign of TA (11). In addition, an intimal dissection in the common carotid artery was identified, which is not typically a unique imaging finding of TA. The patient had a 10-year history of TA, resulting in increased artery stiffness, which may increase the flow velocity of the involved artery. Furthermore, chronic inflammation impelled the lower wall elasticity and thus the intima was vulnerable to denudation. The involved artery segment showed mild stenosis (35\%); however, the PSV was markedly accelerated $(305 \mathrm{~cm} / \mathrm{s})$, indicating severe stenosis. The high PSV observed in the current patient may have resulted from increased arterial stiffness and decreased elasticity.

The pathophysiology of dissection remains poorly understood. A number of studies have suggested that patients with artery dissection may have a genetically-determined weakness of the vessel wall, and that environmental factors, such as acute infection or minor trauma, may serve as triggers (12-14). The intimal dissection in the common carotid artery may be associated with chronic inflammation of the vessel wall, vulnerable intima and hyperdynamic flow. This mechanism of chronic inflammation may result in a moving intimal flap in the involved artery, which is another unique character of intimal injury (15). In patients with an advanced stage of TA, intimal defects may rarely occur as a result of stenosis, which can cause insufficient blood flow to downstream vessels and decreased blood flow velocity. Therefore, whether arterial intima defects are unique to TA requires further study.

In conclusion, as one of the primary causes of ischemic stroke in young adults, artery dissection requires early identification and management (15). Accordingly, regular follow-up is necessary for patients with chronic TA, and strategies for the prevention of cerebrovascular ischemia should be carefully designed when an artery dissection is identified. Artery dissection in the common carotid artery is a rare phenomena for patients with TA, and the exact mechanism is not known, but is one of the as one of the primary causes of ischaemic stroke in young adults. Further research is required in order to improve the understanding of the underlying pathophysiology of TA accompanied by common carotid artery dissection, to assess the long-term outcome, and to provide treatment and prevention strategies.

\section{References}

1. Sadurska E, Jawniak R, Majewski M and Czekajska-Chehab E: Takayasu arteritis as a cause of arterial hypertension. Case report and literature review. Eur J Pediatr 171: 863-869, 2012.

2. Maffei S, Di Renzo M, Bova G, Auteri A and Pasqui AL: Takayasu's arteritis: A review of the literature. Intern Emerg Med 1: 105-112, 2006

3. Hotchi M: Pathological studies on Takayasu arteritis. Heart Vessels Suppl 7: 11-17, 1992.

4. Meini S, De Franco V, Auteri A and Pieragalli D: Images in cardiovascular medicine. Takayasu's arteritis: The 'macaroni sign'. Circulation 114: e544, 2006.

5. Khalife T, Alsac JM, Lambert M, Messas E, Duong Van Huyen JP, Bruneval P, Farahmand P, Julia P and Fabiani JN: Diagnosis and surgical treatment of a Takayasu disease on an abdominal aortic dissection. Ann Vasc Surg 25: 556.e1-556.e5, 2011.

6. Gaul C, Dietrich W, Friedrich I, Sirch J and Erbguth FJ: Neurological symptoms in type A aortic dissections. Stroke 38: 292-297, 2007.

7. Wildburg G, Zahn R, Zander M, Schleiffer T and Senges J: Takayasu arteritis - a rare differential diagnosis in aortic dissection. A case report. Z Kardiol 84: 1033-1038, 1995.

8. Direskeneli H, Aydin SZ and Merkel PA: Assessment of disease activity and progression in Takayasu's arteritis. Clin Exp Rheumatol 29 (1 Suppl 64): S86-S91, 2011.

9. Vaideeswar P and Deshpande JR: Pathology of Takayasu arteritis. A brief review. Ann Pediatr Card 6: 52-58, 2013.

10. Andrews J and Mason JC: Takayasu's arteritis-recent advances in imaging offer promise. Rheumatology (Oxford) 46: 6-15, 2007.

11. Maeda H, Handa N, Matsumoto M, Hougaku H, Ogawa S, Oku N, Itoh T, Moriwaki H, Yoneda S and Kimura K: Carotid lesions detected by B-mode ultrasonography in Takayasu's arteritis: 'Macaroni sign' as an indicator of the disease. Ultrasound Med Biol 17: 695-701, 1991

12. Brandt T, Orberk E, Weber R, Werner I, Busse O, Müller BT, Wigger F, Grau A, Grond-Ginsbach C and Hausser I: Pathogenesis of cervical artery dissections: Association with connective tissue abnormalities. Neurology 57: 24-30, 2001.

13. Schievink WI: Spontaneous dissection of the carotid and vertebral arteries. N Engl J Med 344: 898-906, 2001.

14. Debette S and Leys D: Cervical-artery dissections: Predisposing factors, diagnosis and outcome. Lancet Neurol 8: 668-678, 2009.

15. Yang KK and Park JK: Chronic Takayasu arteritis with a multifocal intimal defect and an intimal flap in them common carotid artery. J Ultrasound Med 32: 2217-2219, 2013. 